\title{
Influence of Fibrinogen Deficiency on Clot Formation in Flow by Hybrid Model
}

\author{
A. Tosenberger ${ }^{1,2 *}, \mathrm{~N}$. Bessonov $^{3}, \mathrm{~V}$. Volpert $^{1,2}$ \\ ${ }^{1}$ Institut Camille Jordan, UMR 5208 CNRS, University Lyon 1, 69622 Villeurbanne, France \\ ${ }^{2}$ INRIA Team Dracula, INRIA Antenne Lyon la Doua, 69603 Villeurbanne, France \\ ${ }^{3}$ Institute of Mechanical Engineering Problems, 199178 Saint Petersburg, Russia
}

\begin{abstract}
In this work we develop the 2D model suggested in [32] in order to study the impact of fibrinogen concentration and the fibrin polymer production rate on clot growth in flow. The model is based on the method of Dissipative Particle Dynamics describing blood plasma flow and platelet suspension and on a system of partial differential equations describing blood coagulation regulatory network. We study the influence of parameters on clot development and on its final size.
\end{abstract}

Keywords and phrases: blood coagulation, clot growth, hybrid models, Dissipative Particle Dynamics

Mathematics Subject Classification: 74F10, 92C35, 92C50

\section{Introduction}

Blood coagulation in flow has been extensively studied. However, there are still many open questions concerning clot growth. This sensitive and crucial mechanism by which the body in higher animals prevents blood loss is closely related to many blood disorders, i.e. it either affects them or is being affected by them. In attempts to shed light on the underlying biological, chemical and mechanical processes, mathematical modelling is becoming increasingly important.

There are three main groups of modelling approaches in studying of blood coagulation: continuous, discrete and hybrid models. In continuous models $[2,4,5,8,16,30]$ the concentrations of proteins, so-called blood factors, are described by systems of partial differential equations. All the reactions between blood factors in the process of blood coagulation form a very complex regulatory network, generating a large systems of PDEs. Such models are also able to accurately describe the diffusion of proteins in the flow. However, continuous models do not consider interactions between blood cells, but rather describe them also in terms of concentrations [30]. Contrary to the continuous models, the discrete models $[13,25,29,33-$ 36] focus on the description of individual cells and are thus convenient for studying their interactions in flow. Where they come short is in describing the diffusion of blood factors in the flow. For this reason the third type of approaches was developed - the so-called hybrid models. Hybrid models combine continuous and discrete methods in order to describe different aspects of a studied phenomenon. In this sense they

*Corresponding author. E-mail: tosenberger@math.univ-lyon1.fr 
are often considered in terms of multi-scale modelling. In the last decade several different hybrid models of blood coagulation in flows were developed [12, 13, 25, 29, 33-36].

In our approach to modelling of blood coagulation in flow we have developed a 2D hybrid model in which a discrete simulation method called Dissipative Particle Dynamics (DPD) is used to model the suspension of platelets in the plasma flow. The continuous part of the model consists of a system of partial differential equations describing the concentrations of proteins in the flow and the corresponding regulatory network. The model is based on several important biological assumptions which have been experimentally confirmed. First, we assume that platelet aggregation may precede platelet activation $[9,17-20,24,37]$. This assumption is crucial for faster flows (arterioles, venules) where the initial platelet aggregate protects protein concentrations from the flow, allowing for the bio-chemical reactions to occur at the injury site [28]. The second assumption is that the end product of the coagulation reactions, fibrin polymer, forms a net which reinforces the platelet aggregate. In the third assumption, platelets which are covered by fibrin polymer become non-adhesive [21], and thus cannot attach new platelets from the flow. As a result of this model we have proposed a new possible mechanism of clot growth and growth arrest in flow in normal healthy conditions [32]. We will measure the total concentrations of the three proteins included in the model (thrombin, fibrinogen, fibrin polymer) and their variations for different values of parameters.

\section{Model description}

The 2D hybrid model developed in this work uses the Dissipative Particle Dynamics (DPD) method to describe the flow of platelet suspension in blood plasma. Concentrations of proteins in the blood and their diffusion and reactions in the flow are modelled by a system of partial differential equations. Finally, platelet aggregation is modelled as an interaction between individual platelets in which they form elastic bonds.

\subsection{Flow model}

The flow of blood plasma and the suspension of platelets is modelled by DPD. It is a well known simulation method convenient for modelling hydrodynamics of complex fluids, such as blood $[6,7,10,11,15,22,26,27]$. As a mesoscale method, the particles represent small drops of fluid, rather than individual atoms or molecules. Between each two particles in some $r_{c}>0$ proximity there exist three pair-wise forces conservative, dissipative and random force:

$$
\begin{aligned}
\mathbf{F}_{i j}^{C} & =F_{i j}^{C}\left(r_{i j}\right) \hat{\mathbf{r}}_{i j}, \\
\mathbf{F}_{i j}^{D} & =-\gamma \omega^{D}\left(r_{i j}\right)\left(\mathbf{v}_{i j} \cdot \hat{\mathbf{r}}_{i j}\right) \hat{\mathbf{r}}_{i j} \\
\mathbf{F}_{i j}^{R} & =\sigma \omega^{R}\left(r_{i j}\right) \frac{\xi_{i j}}{\sqrt{d t}} \hat{\mathbf{r}}_{i j},
\end{aligned}
$$

where $\mathbf{r}_{i}$ is the vector of position of the particle $i, \mathbf{r}_{i j}=\mathbf{r}_{i}-\mathbf{r}_{j}, r_{i j}=\left|\mathbf{r}_{i j}\right|, \hat{\mathbf{r}}_{i j}=\mathbf{r}_{i j} / r_{i j}$, and $\mathbf{v}_{i j}=\mathbf{v}_{i}-\mathbf{v}_{j}$ is the difference between the velocities of two particles, $\gamma$ and $\sigma$ are coefficients which determine the strength of the dissipative and the random force respectively, while $\omega^{D}$ and $\omega^{R}$ are weight functions; $\xi_{i j}$ is a normally distributed random variable with zero mean, unit variance, and $\xi_{i j}=\xi_{j i}$. The conservative force is given by the equality

$$
F_{i j}^{C}\left(r_{i j}\right)= \begin{cases}a_{i j}\left(1-r_{i j} / r_{c}\right) & \text { for } r_{i j} \leq r_{c} \\ 0 & \text { for } r_{i j}>r_{c}\end{cases}
$$

where $a_{i j}$ is the conservative force coefficient between particles $i$ and $j$, and $r_{c}$ is the cut-off radius. 
The random and dissipative forces form a thermostat. If the following two relations are satisfied, the system will preserve its energy and maintain the equilibrium temperature:

$$
\omega^{D}\left(r_{i j}\right)=\left[\omega^{R}\left(r_{i j}\right)\right]^{2}, \quad \sigma^{2}=2 \gamma k_{B} T,
$$

where $k_{B}$ is the Boltzmann constant and $T$ is the temperature. The weight functions are determined by:

$$
\omega^{R}\left(r_{i j}\right)= \begin{cases}\left(1-r_{i j} / r_{c}\right)^{k} & \text { for } r_{i j} \leq r_{c} \\ 0 & \text { for } r_{i j}>r_{c}\end{cases}
$$

where $k=1$ for the original DPD method, but it can be also varied in order to change the dynamic viscosity of the simulated fluid [10]. The motion of particles is determined by Newton's second law of motion:

$$
d \mathbf{r}_{i}=\mathbf{v}_{i} d t, \quad d \mathbf{v}_{i}=\frac{d t}{m_{i}} \sum_{j \neq i}\left(\mathbf{F}_{i j}^{C}+\mathbf{F}_{i j}^{D}+\mathbf{F}_{i j}^{R}\right),
$$

where $m_{i}$ is the mass of the particle $i$.

The simplest way to integrate the equations of motion (2.7) is by use of Euler method. However, more refined methods, like modified velocity-Verlet method [3,15], can be used, which allows a certain increase in the time step $d t$ (by the factor of about 50 [15]). Additionally, the relative velocity term, $\mathbf{v}_{i j}=\mathbf{v}_{i}-\mathbf{v}_{j}$, in equation (2.2) can be integrated in a semi-implicit way - as $\mathbf{v}_{i}^{n}-\mathbf{v}_{j}^{n-1}$, where $n$ denotes a current time step. The last integration method allows further increase in time step, however it is not conservative. For this reason it is preferable to verify the obtained results by comparison to results obtained by one of the first two methods which are conservative. In this work we have used the last method in combination with the Euler method.

In scope of this work a flow in a vessel of $50 \mu \mathrm{m}$ in diameter was simulated by 4500 particles with the DPD time step of $10^{-5} \mathrm{~s}$. As hydrodynamic properties of blood plasma are close to those of water, the density and viscosity of the simulated medium were set to $1000 \mathrm{~kg} / \mathrm{m}^{3}$ and $1.24 \mathrm{mPa} \cdot \mathrm{s}$, respectively. The mean velocity of the flow in simulations was $18.5 \mathrm{~mm} / \mathrm{s}$, which corresponds to the wall shear rate of 1500 $\mathrm{s}^{-1}$.

\subsection{Regulatory network model}

The coagulation regulatory network is modelled by a system of partial differential equations. We consider a minimal scheme of the regulatory network, as shown in Figure 1, which takes into account the main characteristics of blood coagulation cascade: the initial local activation of prothrombin to thrombin at the injury site, the self-accelerated thrombin production, the production of fibrin from fibrinogen in the presence of thrombin as an enzyme, and finally the production of fibrin polymer which forms a fibrin net. The reactions represented in Figure 1 are modelled by the following system of equations:

$$
\begin{aligned}
\frac{d T_{h}}{d t}+\mathbf{v} \cdot \nabla T_{h} & =D_{T_{h}} \Delta T_{h}+k_{1}\left(T_{h}\right)\left(C_{T_{h}}-T_{h}\right)-\lambda T_{h}, \\
\frac{\partial F_{g}}{\partial t}+\mathbf{v} \cdot \nabla F_{g} & =D_{F_{g}} \Delta F_{g}-k_{3}\left(T_{h}\right) F_{g}\left(C_{F_{p}}-F_{p}\right), \\
\frac{\partial F_{p}}{\partial t} & =k_{3}\left(T_{h}\right) F_{g}\left(C_{F_{p}}-F_{p}\right),
\end{aligned}
$$


where $T_{h}, F_{g}$ and $F_{p}$ are thrombin, fibrinogen and fibrin polymer concentrations respectively, $\mathbf{v}$ is the velocity field obtained form the DPD simulation of blood plasma and platelets, $D_{T_{h}}$ and $D_{F_{g}}$ are diffusion coefficients for thrombin and fibrinogen respectively, $C_{T_{h}}$ is the initial prothrombin level, $\lambda$ is the thrombin degradation constant, and $C_{F_{p}}$ is the maximum local concentration of fibrin polymer due to saturation. The functions $k_{1}\left(T_{h}\right)$ and $k_{3}\left(T_{h}\right)$ are reaction rates for the production of thrombin and fibrin polymer respectively, and they are defined as follows:

$$
k_{1}\left(T_{h}\right)=k_{1}^{0} \frac{T_{h}^{2}}{T_{0}+T_{h}}, \quad k_{3}\left(T_{h}\right)=k_{3}^{0} T_{h}
$$

where $k_{1}^{0}, T_{0}$ and $k_{3}^{0}$ are some constants. The form of the reaction rate for thrombin $k_{1}\left(T_{h}\right)$ is chosen as in equation (2.9) in order to have a bistable system, with one stable state in $T_{h}^{(1)}=0$, representing the state with no production of thrombin (and thus no coagulation), and the other one in $0<T_{h}^{(2)}<C_{T_{h}}$, representing the sustainable production of thrombin. Such bistable kinetics corresponds to biological observations.

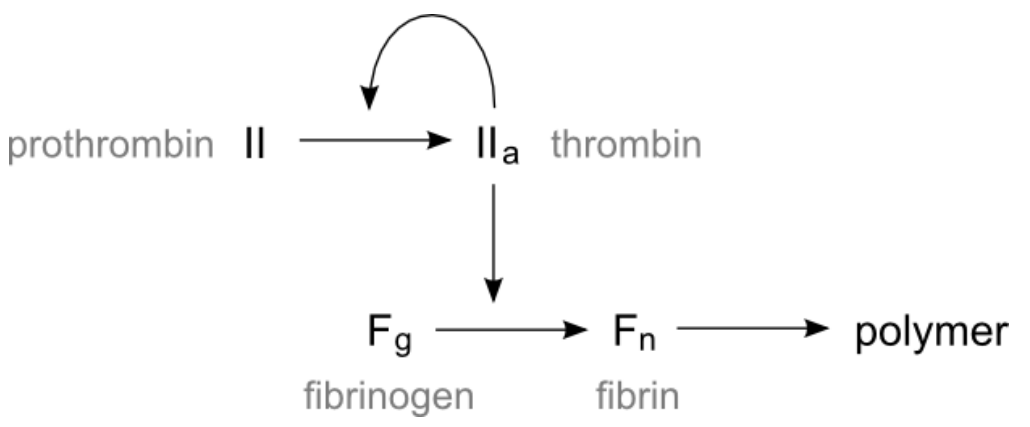

Figure 1. Blood coagulation pathway, as described in the model. The model takes into account the local production of thrombin due to the presence of tissue factor at the injury site, the self-accelerated thrombin production, and the fibrin polymer production from fibrinogen in the presence of thrombin as an enzyme.

The initial and boundary conditions for thrombin correspond to a small initial production of thrombin at the injury site due to the presence of tissue factor:

$$
\begin{aligned}
\left.\frac{\partial T_{h}}{\partial x}(x, y, t)\right|_{x=0, L} & =\left.\frac{\partial T_{h}}{\partial y}(x, y, t)\right|_{y=0, D}=0 \\
\left.T_{h}(x, y, t)\right|_{t=0} & = \begin{cases}1, & \text { if } x \in\left[w_{B}, w_{E}\right] \text { and } y=0, \\
0, & \text { if } x \notin\left[w_{B}, w_{E}\right] \text { or } y \neq 0,\end{cases}
\end{aligned}
$$

where $[0, L] \times[0, D]$ is the simulation domain, i.e. a part of the blood vessel with the length $L$ and diameter $D$, and where $\left[w_{B}, w_{E}\right] \times\{0\}$ is the part of the domain representing the vessel injury site. On the other hand the initial and boundary conditions for fibrinogen represent its constant presence in the blood:

$$
\begin{gathered}
\left.\frac{\partial F_{g}}{\partial x}(x, y, t)\right|_{x=L}=\left.\frac{\partial F_{g}}{\partial y}(x, y, t)\right|_{y=0, D}=0, \\
\left.F_{g}(x, y, t)\right|_{x=0}=F_{g}^{0},\left.\quad F_{g}(x, y, t)\right|_{t=0}=F_{g}^{0} .
\end{gathered}
$$


The equation for fibrin polymer concentration does not contain the advection and diffusion terms since fibrin polymers form the fibrin net which cannot diffuse or flow.

In order to numerically solve equations (2.8) Alternating Direction Implicit (ADI) method was used. Diffusion terms was approximated by second order central finite difference scheme, while first order finite difference upwind scheme was used to approximate advection terms. Although the regulatory network model is phenomenological, some of values of parameters in equations (2.8), like diffusion coefficients $D_{T_{h}}$ and $D_{F_{g}}$, were taken from the more detailed model [23] which describes blood coagulation in a quiescent medium. The velocity in the discrete (DPD) and continuous (PDEs) parts of the model is considered in different reference frames - Lagrangian and Eulerian. In order to pass from one to another velocities of particles are measured in DPD on a fixed spatial subdivision of the simulation domain. Additionally, the measurements are done during short periods, after which the velocity field is obtained by calculating the mean velocity for each element of the spatial subdivision. The measured velocity field is in Eulerian reference frame and enters the equations (2.8) via advection terms.

\subsection{Platelet aggregation}

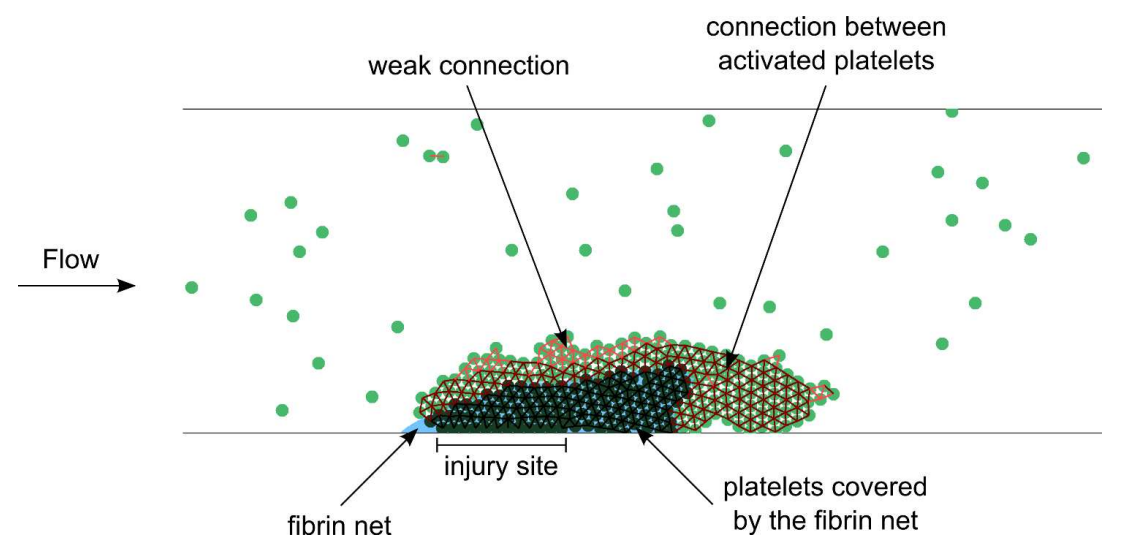

Figure 2. A snapshot from a simulation of clot growth with the hybrid model. Green particles show platelets, while dark green are platelets covered by the fibrin net. The fibrin net is denoted by the blue colour. The lines between platelets show the corresponding connections and their types: light red for the weak aggregate, dark red for activated platelets and black for platelets covered in fibrin polymer.

In the model platelets are considered as single DPD particles, thus the uniform diameter of all particles, for both plasma and platelets, was set to correspond to the approximate physical diameter of platelets $(2 \mu \mathrm{m})$. The concentration of platelets in simulations was set to $3.672 \cdot 10^{12} \mathrm{~L}^{-1}$, which is about 10 times higher than the normal concentration of platelets in blood $\left(0.15-0.4 \cdot 10^{12} L^{-1}\right)$. This is done for two reasons. Firstly, the spatial distribution of platelets in simulations is uniform, and, as there are no erythrocytes in the flow, platelets do not migrate to the vessel wall. In presence of erythrocytes platelets are being pushed closer to the vessel wall, thus greatly increasing their concentration in the near wall region where the clot is formed [7]. Secondly, by increasing the platelet concentration the clot will grow faster, thus reducing the time necessary to simulate the clot growth and arrest. Due to elastic properties of the blood clot, the model of platelet aggregation is based on Hooke's law. In case of a physical contact between two platelets a spring connection between them is established:

$$
\mathbf{F}_{i j}^{A}=f^{A}\left(t_{i j}\right)\left(1-\frac{r_{i j}}{d_{C}}\right) \hat{\mathbf{r}}_{i j}
$$


where $d_{C}$ is a critical distance after which the connection breaks, $t_{i j}$ is the age of the connection, and $f^{A}\left(t_{i j}\right)$ is a function of the connection strength given by:

$$
f^{A}\left(t_{i j}\right)= \begin{cases}f_{1}^{A} & \text { if } F_{p}(i) \text { or } F_{p}(j)<c_{F_{p}}, \quad \text { and } \quad t_{i j}<t_{c} \\ f_{2}^{A} & \text { if } F_{p}(i) \text { or } F_{p}(j)<c_{F_{p}}, \quad \text { and } \quad t_{i j} \geq t_{c} \\ f_{3}^{A} & \text { if } F_{p}(i) \text { and } F_{p}(j) \geq c_{F_{p}},\end{cases}
$$

where $f_{1}^{A}<f_{2}^{A}<f_{3}^{A}$ are the three strengths of inter-platelet connections, representing respectively a weak bond due to GPIb receptors, a medium bond due to platelet activation and a strong bond due to the reinforcement by the fibrin polymer net. Next, $t_{c}$ is the time needed for platelet activation measured from the moment when the connection was established, $F_{p}(i)$ and $F_{p}(j)$ is the concentration of fibrin polymer $F_{p}$ at the positions of particles $i$ and $j$ respectively, $c_{F_{p}}$ is the critical level of fibrin polymer. A platelet is considered to be covered by the fibrin net if the fibrin polymer concentration becomes larger than $c_{F_{p}}$ at the position of that platelet. Such platelets are adhesion resistant [21] and cannot attach new platelets from the flow. The choice of step function (equation (2.15)) for modelling platelet activation (depending on $t_{c}$ ) is explained in $[31,32]$.

The DPD model of the flow (Section 2.1) and the platelet aggregation model (Section 2.3) joined with the regulatory network model (Section 2.2) make a hybrid (discrete-continuous) model of blood coagulation. Both the discrete and the continuous part influence each other. On the one hand the discrete part of the model (DPD) together with platelet aggregation at the injury site influences the concentrations of blood factors via advective terms in equations (2.8). On the other hand the concentration of fibrin polymer influences the stability of the growing platelet clot and thus indirectly changes the flow properties.

\section{Results}

The process of clot growth simulated with this model can be described as follows: a) the initial weak aggregate is formed at the injury site $(0-1 \mathrm{~s}), \mathrm{b})$ it is reinforced by platelet activation (1-2 s), c) the decreased flow speed inside of the platelet aggregate enables the accumulation of protein concentrations and the beginning of bio-chemical reactions which result in the formation of the fibrin net $(2-7 \mathrm{~s}), \mathrm{d})$ the fibrin net reinforces the clot and allows its further growth $(7-14 \mathrm{~s}), \mathrm{e})$ the growing clot narrows the blood vessel, increasing the flow pressure on the clot, which results in a breaking of its weaker outer parts (14-30 s), f) after several detachments of outer parts only the stable part of the clot, covered by fibrin polymer, is left at the injury site $(30 \mathrm{~s}), \mathrm{g})$ due to the non-adhesive effect of fibrin polymer, the remaining clot cannot attach new platelets from the flow, thus it stops growing. The time spans of clot growth stages (given in the brackets) are obtained from a single simulation. In that simulation the total time of clot growth was about 30 seconds, however this value can vary depending on the values of simulation parameters. The described mechanism of clot growth was suggested in [32] where a simplified model of blood coagulation reactions described by a single reaction-diffusion equation was considered. In this work we observe it and study for a more complete model of blood coagulation described by three reaction-diffusion equations. A snapshot from a simulation is given in Figure 2, showing a clot structure in the stage d) of the described clot growth. Figure 3 shows distributions of velocity and protein concentrations at the final stage of the clot growth.

The described process of clot formation depends on the interaction between the platelet aggregate and protein reactions in the flow. Without any of them the clot is unable to form in faster flows (arterioles, venules). In this work we observe the total concentrations of simulated blood factors (thrombin, fibrinogen and fibrin polymer) and we study the impact of the most important parameters. For this purpose we simulated blood flow in a channel of $50 \mu \mathrm{m}$ in diameter with average flow speed of $12.5 \mathrm{~mm} / \mathrm{s}$. The values of strength coefficients of platelet bonds were set to $f_{1}^{A}=0.4 n \mathrm{~N}, f_{2}^{A}=0.8 n \mathrm{~N}, f_{3}^{A}=10 n \mathrm{~N}$, with the platelet activation time $t_{c}=0.3 \mathrm{~s}$. The strengths of inter-platelet bonds are in a similar range as observed by a discrete model of platelet aggregation described in [25]. For the basic simulation the 
a) $\mathbf{v x}$

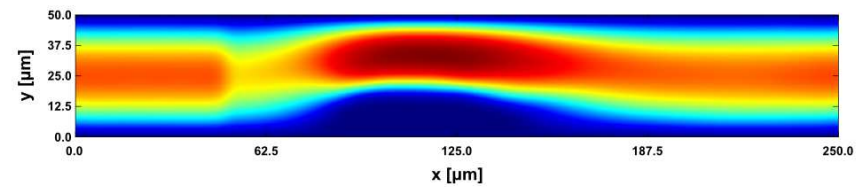

b) $v_{y}$

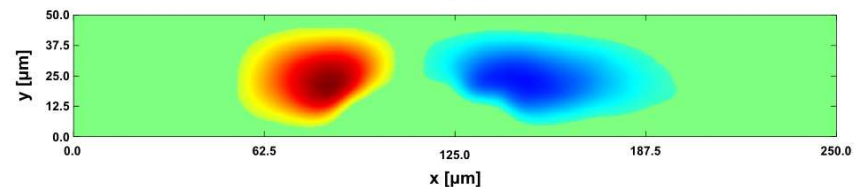

c) $\mathbf{T}$

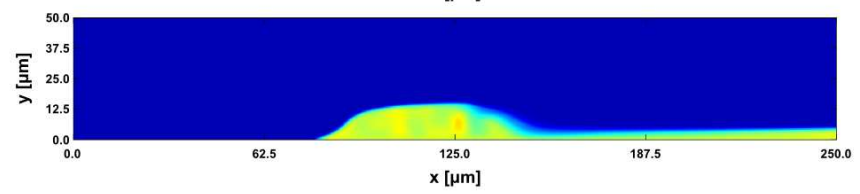

d) $F_{g}$

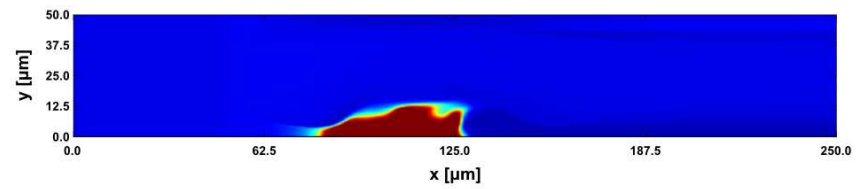

e) $F_{p}$
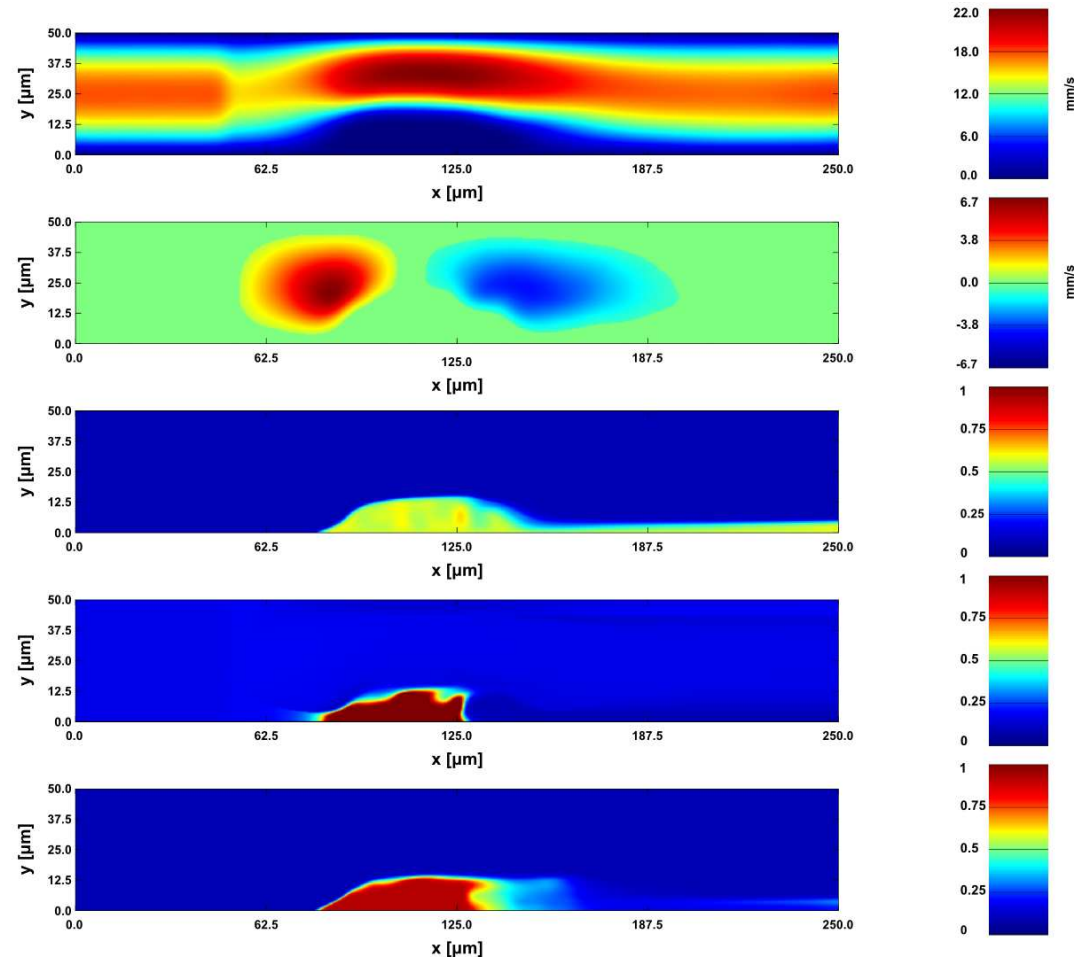

Figure 3. Velocity and concentrations profiles at the fully developed clot stage: a) distribution of the component of velocity parallel to the flow axis, b) distribution of the component of velocity perpendicular to the flow axis, c) thrombin distribution, d) fibrinogen distribution, e) fibrin polymer distribution. The flow is from left to right.

values of PDEs' parameters were set to the following: diffusion coefficients $D_{T_{h}}, D_{F_{g}}=0.003 \mathrm{~mm}^{2} / \mathrm{min}_{\text {, }}$ thrombin degradation rate constant $\lambda=1.2 \cdot 10^{4} \mathrm{~min}^{-1}$, thrombin and fibrin polymer production rate constants $k_{1}^{0}=3.3 \cdot 10^{4} \mathrm{~min}^{-1}$ and $k_{3}^{0}=6 \mathrm{~min}^{-1}$ respectively, while the concetrations were considered in the non-dimensional units $T_{0}=0.1$, initial prothrombin level $C_{T_{h}}=1$, maximum local concentration of fibrin polymer $C_{F_{p}}=1$, and initial level of fibrinogen $F_{g}^{0}=0.1$.

In Figure 4 the clot size and the total concentrations of thrombin, fibrinogen and fibrin polymer in time are shown for the basic simulation. The value of a total concentration is obtained by integration over the whole simulation domain. The clot size, measured in the number of platelets (red dashed curve), shows the moments of the detachments of the weaker outer parts of the clot due to the increased pressure from the flow on the clot. At the end clot stops growing in size. The other three curves denote total concentrations in the simulation area of thrombin (blue), fibrinogen (cyan) and fibrin polymer (green). The thrombin concentration at first grows due to the initial concentration produced at the injury site and the presence of the tissue factor, and it continues to grow until it fills the whole platelet aggregate. The concentration which diffuses outside of the clot is taken away by the flow. Thus the total thrombin concentration remains limited by the clot size, and it is suddenly reduced when some parts of the clot detach. On the other hand fibrinogen is constantly present in the flow and it accumulates inside of the clot by inflow and diffusion. In presence of thrombin fibrinogen is consumed in the process of the production of fibrin polymer. Finally, the fibrin polymer concentration rises inside of the growing clot but is being limited by its size as thrombin is present only inside of the clot. Sudden changes in the growth rate of fibrin polymer are noticeable at the moments of the detachments of the outer parts of the clot. 


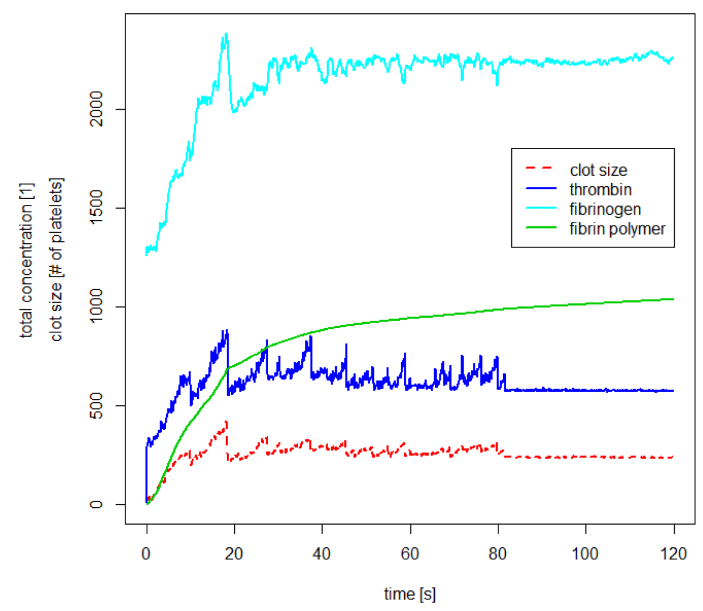

FiguRE 4. Results of the basic simulation: a) clot size (red dashed) in time expressed in the number of platelets, b) total thrombin concentration (blue solid), c) total fibrinogen concentration (cyan solid), and d) total fibrin polymer concentration (green solid) expressed in non-dimensional units. Note that the clot size and concentration values are drawn on the same scale. However, for the clot size curve (red dashed) the scale denotes the total number of platelets in the clot, while for thrombin, fibrinogen and fibrin polymer curves the scale denotes the total concentration of the respective blood factor (expressed in non-dimensional units).

Physiologically, there exist three types of fibrinogen deficiency [1]: dysfibrinogenemia, hypofibrogenemia and afibrinogenemia. In the case of dysfibrinogenemia the level of fibrinogen in blood is normal (1.5-3.5 g/L), however the fibrinogen does not function properly due to genetic mutations. Depending on the type of mutation dysfibrinogenemia can be either asymptomatic, or can cause bleeding or thrombosis. Hypofibrogenemia is a deficiency which manifests in lower than normal level of fibrinogen in blood (0.1-1.5 $\mathrm{g} / \mathrm{L})$. As the level of fibrinogen is lower, the condition will lead to more bleeding. Finally, afibrinogenemia denotes a complete lack of fibrinogen in blood $(<0.1 \mathrm{~g} / \mathrm{L})$, which causes serious bleeding.

Using the described model we first study the influence of the initial fibrinogen concentration $\left(F_{g}^{0}\right)$ on the clot formation. Figure 5 shows the clot size and the total concentration of blood factors at different initial fibrinogen concentrations, ranging from 0.01 to 0.5 . At the beginning (the first 15 seconds) the clot size increases with approximately the same rate in all simulations except the one with $F_{g}^{0}=0.01$. However, the higher level of fibrinogen results in a faster fibrin polymer production, thus allowing the clot to grow larger. Thrombin concentrations also remain similar in the first 15 seconds, reflecting the sizes of the corresponding clots. Fibringen concentrations rise due to the higher initial level and its accumulation in the clot. Finally, the fibrin polymer concentration growth rate is at first increased due to the increased initial fibrinogen concentrations, while later on it becomes limited by the clot size. For the initial level of fibrinogen of 0.01 the production of fibrin polymer is too low to support the growing platelet aggregate. The platelet clot breaks off, and leaves the injury site insufficiently protected. These results qualitatively correspond to the cases of normal, low (hypofibrogenemia) and too low (afibrinogenemia) levels of fibrinogen in blood.

Next, we vary the reaction rate constant of fibrin polymer production. Figure 6 shows the clot size and total concentration of blood factors for values of $k_{3}^{0}$ ranging from 0.6 to $30 \mathrm{~min}^{-1}$. For low rates of fibrin polymer production (here 0.6 to $3 \mathrm{~min}^{-1}$ ) the clot is unable to form as its critical size is achieved before it is supported by the fibrin net, which results in the complete detachment of the initial platelet 
a)

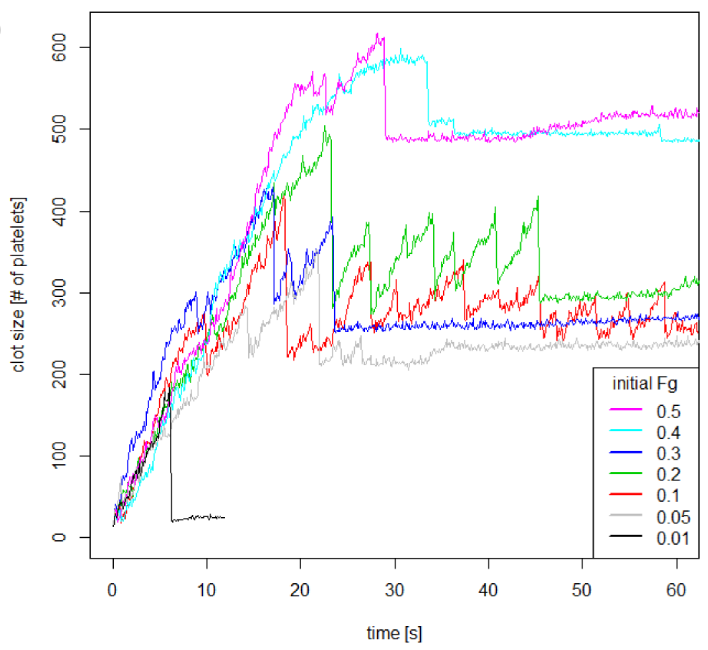

c)

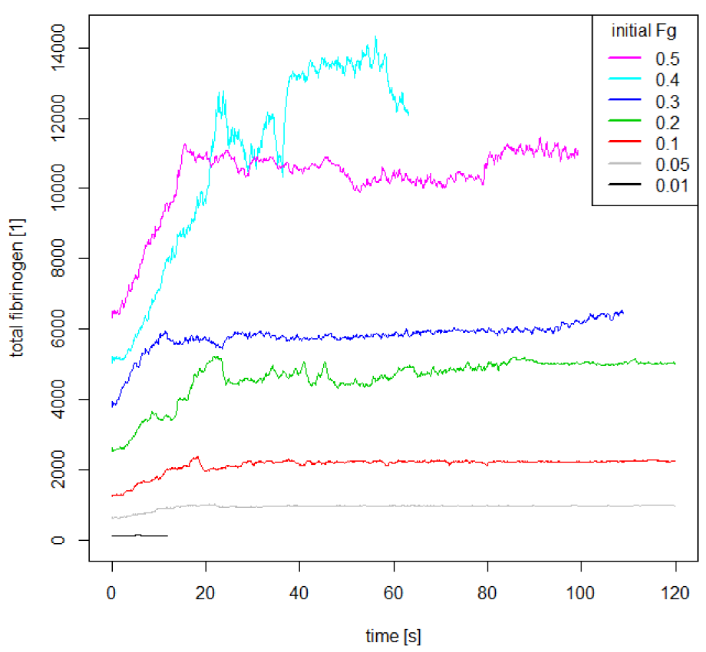

b)

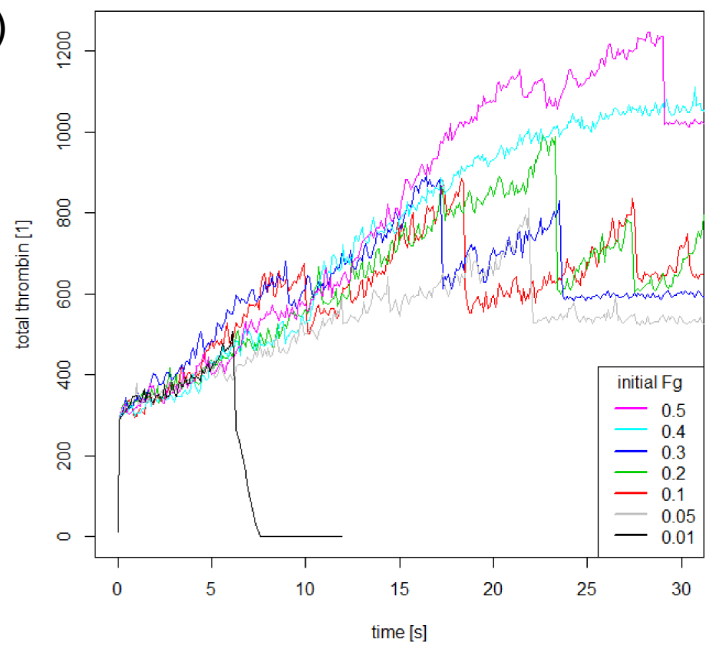

d)

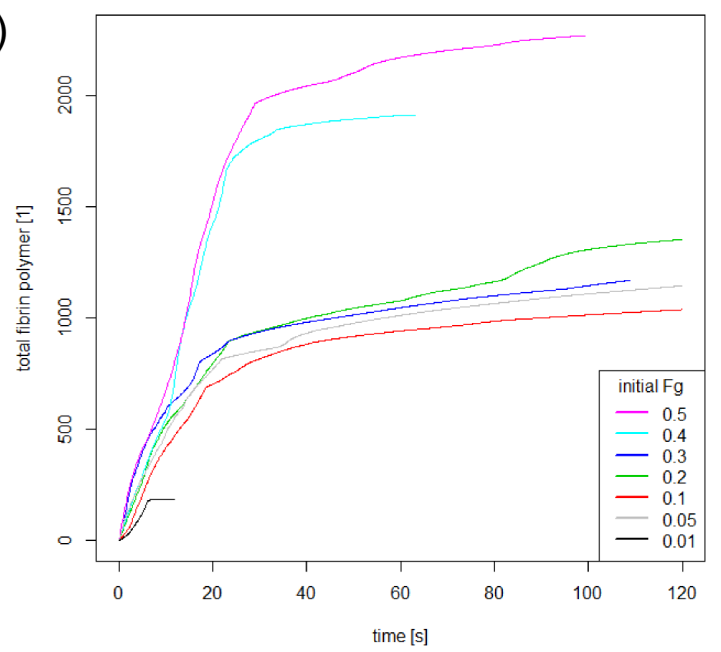

FiguRE 5. Variation of the initial fibrinogen level $F_{g}^{0}$ from 0.01 to 0.5 : a) clot size in time, b) total thrombin concentration in time, c) total fibrinogen concentration in time, d) total fibrin polymer concentration in time.

aggregate and the removal of the thrombin concentration by the flow. As the production rate increases $\left(6,15\right.$ and $\left.30 \mathrm{~min}^{-1}\right)$, the clot grows to a larger size since it is supported by a faster fibrin net generation.

\section{Discussion}

The model presented in this work is a minimal model of clot development in flow, which can capture the platelet aggregate - coagulation pathway interaction. It can be used for further refinements by the introduction of more complete models of blood cells and of the regulatory network. In this work we use it to study the impacts of protein concentrations and reaction rates on the clot development and its final size. Furthermore, the regulatory network is described in a form which is simplified but takes into account the main characteristics of coagulation pathways in vivo: the initial production of thrombin at the injury site due to the presence of tissue factor, the self-accelerated production of thrombin, the 
a)

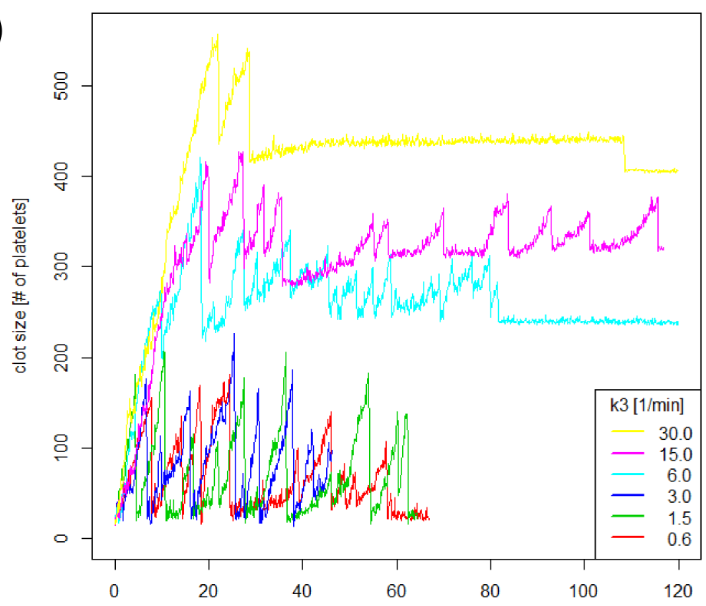

c)

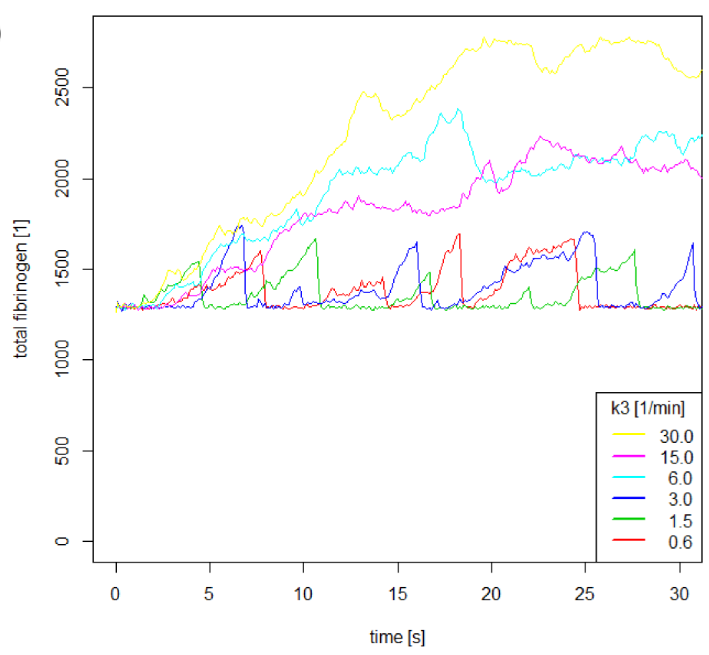

b)

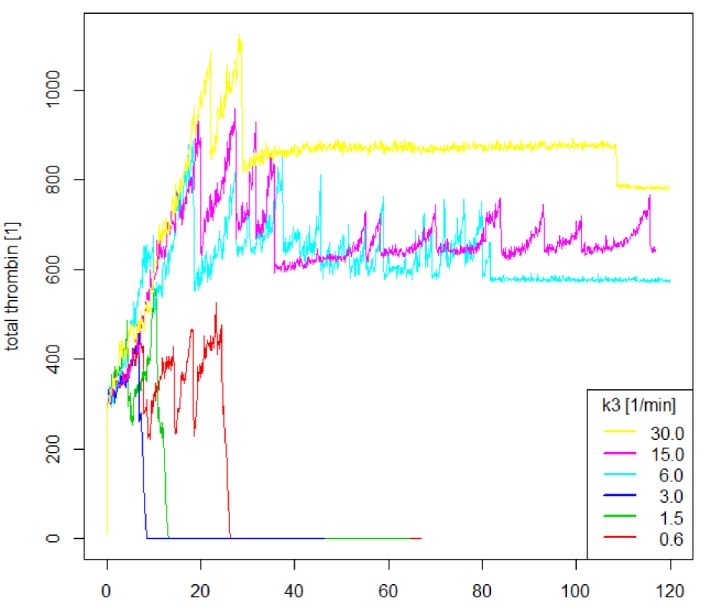

d)

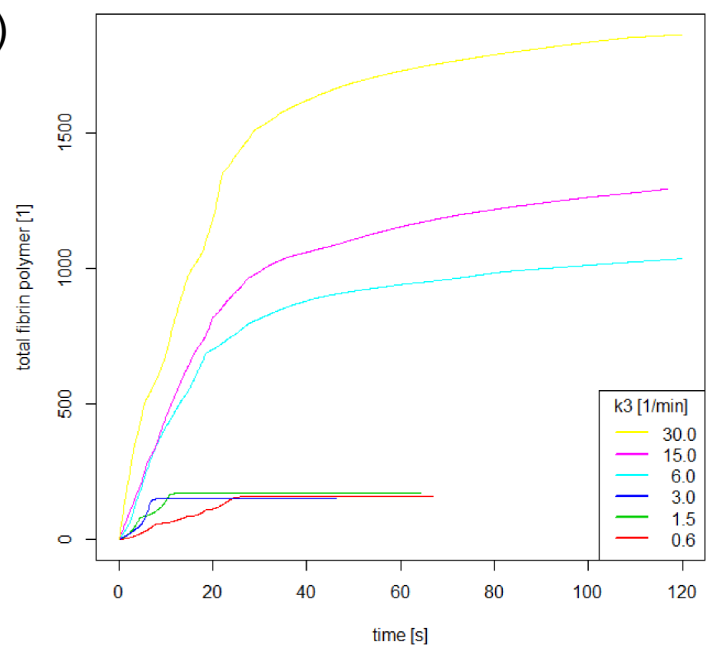

FiguRE 6. Variation of the fibrin polymer production rate coefficient $k_{3}^{0}$ from 0.6 to 30 $\min ^{-1}$ : a) clot size in time, b) total thrombin concentration in time, c) total fibrinogen concentration in time, d) total fibrin polymer concentration in time.

constant presence of fibrinogen in the blood flow and the production of fibrin polymer in the presence of thrombin. Furthermore, the model takes into account the activation of platelets by surrounding aggregated platelets, and the interaction of platelets with fibrin polymer, while other interactions of platelets with the coagulation cascade [14] are omitted. Thus the model is phenomenological and able to produce qualitative rather than quantitative results. As such it serves for studying qualitative aspects of blood coagulation, and we use it here to investigate the influence which the initial fibrinogen level and the fibrin polymer production rate can have on the clot formation in the flow. These results will be used in the upcoming development of a more complete model of clot formation in flow, which will also include a more extensive model of blood coagulation pathways.

The described model is a 2D model, and it takes into account the flow of blood plasma and platelets. Red blood cells and the third dimension are deliberately omitted in order to reduce the complexity of the model and the time necessary to simulate the process of clot growth and arrest. However, the described hybrid model can be extended in order to simulate a 3D flow and/or different types of blood cells. In $[6,7]$ 
the discrete part of the model (DPD) was coupled with a model of erythrocytes and platelets, and used to simulate blood flow in 3D and to study the distribution of the cells in flow. Due to the complexity of the process of clot formation in flow, as well as the extensiveness of the numerical implementations and the computational costs, in the future it will be equally important to continue developing both the $2 \mathrm{D}$ and the 3D model.

Acknowledgements. The work was supported by the research grant ANR "Bimod".

\section{References}

[1] S.S. Acharya, D.M. Dimichele. Rare inherited disorders of fibrinogen. Haemophilia, 14 (2008), 1151-1158.

[2] A.G. Alenitsyn, A.S. Kondratyev, I. Mikhailova, I. Siddique. Mathematical Modeling of Thrombus Growth in Microvessels. Journal of Prime Research in Mathematics, Vol. 4 (2008), 195-205.

[3] M.P. Allen, D.J. Tidesley. Computer Simulation of Liquids. Clarendon, Oxford, 1987.

[4] M. Anand, K. Rajagopal, K.R. Rajagopal. A model for the formation, growth, and lysis of clots in quiescent plasma. A comparison between the effects of antithrombin III deficiency and protein C deficiency. J Theor Biol., 253 (2008), no. 4, 725-38. Epub 2008 Apr 25. PubMed PMID: 18539301.

[5] M. Anand, K. Rajagopal, K.R. Rajagopal. A model for the formation and lysis of blood clots. Pathophysiol Haemost Thromb. 34 2005, no. 2-3, 109-20. PubMed PMID: 16432312.

[6] N. Bessonov, E. Babushkina, S.F. Golovashchenko, A. Tosenberger, F. Ataullakhanov, M. Panteleev, A. Tokarev, V. Volpert. Numerical Modelling of Cell Distribution in Blood Flow. Math. Model. Nat. Phenom., 9 (2014), no. 9, .

[7] N. Bessonov, E. Babushkina, S.F. Golovashchenko, A. Tosenberger, F. Ataullakhanov, M. Panteleev, A. Tokarev, V. Volpert. Numerical Simulations of Blood Flows With Non-uniform Distribution of Erythrocytes and Platelets. Russian Journal of Numerical Analysis and Mathematical Modelling, vol. 28 (2013), no. 5, 443-458.

[8] T. Bodnar, A. Sequeria. Numerical Simulation of the Coagulation Dynamics of Blood. Computational and Mathematical Methods in Medicine, 9 (2008), no. 2, 83-104.

[9] Y.L. Chiu, Y.L. Chou, C.Y. Jen. Platelet deposition onto fibrin-coated surfaces under flow conditions. Blood Cells, 13 (1988), no. 3, 437-50. PubMed PMID: 3382750.

[10] D.A. Fedosov. Multiscale Modeling of Blood Flow and Soft Matter. PhD dissertation at Brown University, (2010).

[11] D.A. Fedosov, I.V. Pivkin, G.E. Karniadakis. Velocity limit in DPD simulations of wall-bounded flows. J. Comp. Phys., 227 (2008) 2540-2559.

[12] N. Filipovic, M. Kojic, A. Tsuda. Modelling Thrombosis Using Dissipative Particle Dynamics Method. Phil. Trans. R. Soc., A 366 2008, 3265-3279.

[13] A.L. Fogelson. Cell-based Models of Blood Clotting. Single-Cell-Based Models in Biology and Medicine (ed. by A.R.A. Anderson, M.A.J. Chaplain, K.A. Rejniak), Mathematics and Biosciences in Interaction, 234-169, Birkhäuser Verlag Basel, 2007.

[14] J.E. Freedman. Molecular Regulation of Platelet-Dependent Thrombosis. Circulation, 112 (2005), 2725-2734.

[15] R.D. Groot, P.B. Warren. Dissipative particle dynamics: Bridging the Gap Between Atomistic and Mesoscopic Simulation. J. Chem. Phys., 107 (1997), no. 11, 4423-4435.

[16] R.D. Guy, A.L. Fogelson, J.P.Keener. Fibrin gel formation in a shear flow. Math Med Biol., 24 (2007), no. 1, 111-30.

[17] S.P. Jackson. The growing complexity of platelet aggregation. Blood, 109 (2007), 227, no. 12, 5087-95. Epub.

[18] C.J. Jen, S.J. Hu, H.J. Wu, T.S. Lin, C.W. Mao. Platelet-fibrin interaction in the suspension and under flow conditions. Adv Exp Med Biol., 281 (1990), 277-85. PubMed PMID: 2102618.

[19] C.J. Jen, J.S. Lin. Direct observation of platelet adhesion to fibrinogen- and fibrin-coated surfaces. Am J Physiol., 261 (1991), (5 Pt 2):H1457-63. PubMed PMID: 1951733.

[20] C.J. Jen, Y.W. Tai. Morphological study of platelet adhesion dynamics under whole blood flow condition. Platelets, 3 (1992), no. 3, 145-53. PubMed PMID: 21043907.

[21] M.M. Kamocka, J. Mu, X. Liu, N. Chen, A. Zollman, B. Sturonas-Brown, K. Dunn, Z. Xu, D.Z. Chen, M.S. Alber, E.D. Rosen. Two-photon intravital imaging of thrombus development. J Biomed Opt, 15 (2010), 016020.

[22] M. Karttunen, I. Vattulainen, A. Lukkarinen. A novel methods in soft matter simulations. Springer, Berlin, 2004.

[23] Y.V. Krasotkina, E.I. Sinauridze, F.I. Ataullakhanov. Spatiotemporal dynamics of fibrin formation and spreading of active thrombin entering non-recalcified plasma by diffusion. Biochimica et Biophysica Acta 1474, (2000), 337-345.

[24] S. Kulkarni, S.M. Dopheide, C.L. Yap, C. Ravanat, M. Freund, P. Mangin, K.A. Heel, A. Street, I.S. Harper, F. Lanza, S.P. Jackson. A revised model of platelet aggregation. J Clin Invest., 105 (2000), no. 6, $783-91$.

[25] I.V. Pivkin, P.D. Richardson, G. Karniadakis. Blood flow velocity effects and role of activation delay time on growth and form of platelet thrombi. PNAS, 103 (2006), 17164-17169.

[26] I.V. Pivkin, P.D. Richardson, G.E. Karniadakis. Effect of Red Blood Cells on Platelet Aggregation. Engineering in Medicine and Biology Magazine, IEEE 28.2 (2009), 32-37.

[27] U.D. Schiller. Dissipative Particle Dynamics. A Study of the Methodological Background. Diploma thesis at Faculty of Physics University of Bielefeld, 2005 
[28] A.M. Shibeko, E.S. Lobanova, M.A. Panteleev, F.I. Ataullakhanov. Blood flow controls coagulation onset via the positive feedback of factor VII activation by factor Xa. BMC Syst Biol, 4 (2010), no. 5.

[29] C.R. Sweet, S. Chatterjee, Z. Xu, K. Bisordi, E.D. Rosen, M. Alber. Modelling platelet - blood flow interaction using the subcellular element Langevin method. J. R. Soc. Interface 8 (2011), 1760-1771.

[30] A. Tokarev, I. Sirakov, G. Panasenko, V. Volpert, E. Shnol, A. Butylin, and F. Ataullakhanov. Continuous mathematical model of platelet thrombus formation in blood flow. Russian Journal of Numerical Analysis and Mathematical Modelling. , 27 (2012), no. 2, 192-212.

[31] A. Tosenberger, F. Ataullakhanov, N. Bessonov, M. Panteleev, A. Tokarev, V. Volpert. Modelling of clot growth and growth stop in flow by the method of dissipative particle dynamics. Russian J. Numer. Anal. Math. Modelling, 27 (2012), no. 5, 507-522.

[32] A. Tosenberger, F. Ataullakhanov, N. Bessonov, M. Panteleev, A. Tokarev, V. Volpert. Modelling of thrombus growth in flow with a DPD-PDE method. Journal of Theoretical Biology, 337 (2013), 0-41.

[33] Z. Xu, N. Chen, M.M. Kamocka, E.D. Rosen, M. Alber. A multiscale model of thrombus development. J. R. Soc. Interface,5 (2008), 705-722.

[34] Z. Xu, J. Lioi, J. Mu, M.M. Kamocka, X. Liu, D.Z. Chen, E.D. Rosen, M. Alber. A Multiscale Model of Venous Thrombus Formation with Surface-Mediated Control of Blood Coagulation Cascade. Biophysical Journal, vol. 98 (2010), 1723-1732.

[35] Z. Xu, N. Chen, S. Shadden, J.E. Marsden, M.M. Kamocka, E.D. Rosen, M. Alber. Study of Blood Flow Impact on Growth of Thrombi Using a Multiscale Model. Soft Matter, 5 (2009), 769-779.

[36] Z. Xu, S. Christleyy, J. Lioiz, O. Kim, C. Harveyx, W. Sun, E.D. Rosen, M. Alber. Multiscale Model of Fibrin Accumulation on the Blood Clot Surface and Platelet Dynamics. Methods in Cell Biology, vol. 110, 2012.

[37] T.N. Zaidi, L.V. McIntire, D.H. Farrell, P. Thiagarajan. Adhesion of platelets to surface-bound fibrinogen under flow. Blood, 88 (1996), no. 8, 2967-72. PubMed PMID: 8874193. 